RESEARCH PAPER

\title{
Characteristics of selectors of nicotine replacement therapy
}

\author{
S Shiffman, M E Di Marino, C T Sweeney
}

Tobacco Control 2005;14:346-355. doi: 10.1136/tc.2004.009183

Objective: To assess differences in demographic and smoking characteristics between smokers who have and have not used nicotine replacement therapy (NRT).

Design: Mail survey of US smokers from a national research panel.

Participants: Smokers 18 years and over who returned a survey on smoking $(n=9630)$. The sample was weighted to match the US smoker population on age and sex.

Main outcome measures: Compared smokers who had/had not used NRT in a quit attempt (ever NRT use

See end of article for authors' affiliations

......................

Correspondence to:

Saul Shiffman, PhD, Pinney

Associates, 201 North

Craig Street, Suite 320

Pittsburgh, PA 15213,

USA; shiffman@

pinneyassociates.com

Received 9 July 2004

Accepted 29 June 2005 or over the counter (OTC) NRT use) on: demographic characteristics, nicotine dependence, history of craving and withdrawal, expected difficulty quitting, and self reported history of smoking related medical illness and psychopathology.

Results: NRT users (both ever-users and OTC users) were more likely to be older, male, and better educated. They were also heavier smokers, had experienced more craving and withdrawal upon quitting, and scored higher on measures of dependence. These differences were evident among light smokers, and remained even when smoking rate and time to first cigarette were controlled.

Conclusion: Smokers who elect to use NRT differ from non-NRT users in ways that predispose them to failure in cessation. Controlling for smoking rate and time to first cigarette does not eliminate these differences, even among light smokers. These differences must be considered when comparing the effectiveness of NRT among samples of smokers who self select their treatment and are likely to bias such outcome comparisons.

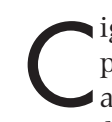
igarette smoking continues to be the greatest source of preventable death and disease in the industrial world, annually killing over 400000 people in the USA alone. ${ }^{1}$ Accordingly, getting smokers to stop smoking is a matter of considerable urgency. Unfortunately, because of nicotine dependence, the vast majority of efforts to stop smoking end in failure; only $3-5 \%$ of untreated smokers achieve prolonged abstinence for 6-12 months after a given quit attempt. ${ }^{2}$ Pharmacological treatment can improve on these success rates; nicotine replacement therapy (NRT) generally doubles success rates. ${ }^{3}$ In the USA, the utilisation of two NRT medications-patch and gum-was increased substantially in 1996 by making them available for sale over the counter (OTC)..$^{4-6}$

While more people are using NRT, we know relatively little about which smokers in particular are electing to use NRT to assist in their quit efforts. In one of the few studies comparing users of NRT with non-users, Cummings and colleagues $^{7}$ found that, compared with non-users, prescription nicotine patch users were more likely to be female, white, and have higher annual household income. Additionally, patch users were more likely to smoke heavily, smoke within 30 minutes after waking (an indicator of dependence ${ }^{8}$ ), report a strong desire to quit smoking, and have made a failed quit attempt in the year before enrolling in the study. This suggests that patch users were more dependent, but more motivated to quit smoking. Understanding who is using NRT may help in understanding the contribution of treatment to cessation and in targeting subgroups that may not be making use of medications. Also, although NRT is effective even among smokers with relatively low nicotine dependence, ${ }^{9}$ studies have shown that it has the largest relative impact on smokers with higher levels of nicotine dependence, because these smokers do so poorly without treatment. ${ }^{10}{ }^{11}$ It seems important to know whether more dependent smokers-or, more generally, those with the greatest difficulty quitting-are in fact self-selecting to use NRT in their quit efforts.

Understanding which smokers elect to use NRT is also important for interpreting findings from epidemiological studies of NRT use. If smokers systematically self-select to use NRT on the basis of dependence and difficulty quitting, then outcome analyses comparing those who do or do not self-select for NRT use will be seriously biased, ${ }^{6}$ because they will be comparing distinct groups with different "baseline" probability of success in quitting. For example, even though NRT efficacy has been proven in hundreds of randomised trials, ${ }^{12}$ retrospective surveys can even yield findings that people who reported using NRT to quit are less likely to have succeeded at quitting. ${ }^{13}$ Pierce and Gilpin ${ }^{6}$ compared cessation rates among self-selected users and non-users of OTC NRT and reported that the differences in outcome were small, especially among lighter smokers. This could be explained if the most dependent smokers-those with the least likelihood of quitting successfully-were being selected or self-selecting to use NRT. Because these high risk groups enter the quit process at the outset with a lower likelihood of success, their quit rates on NRT might not exceed those of lower risk smokers, even if NRT substantially improves their outcomes, making interpretation of outcome comparisons problematic.

The purpose of this paper is to assess differences between current smokers who have and have not used NRT in quitting, using data from a national survey of US smokers. We examined characteristics of smokers who ever used NRT and those who used OTC NRT. Understanding who elects to use NRT in the OTC environment is particularly salient for several reasons. In the OTC setting, smokers can self-select to use or not use NRT without input from a health professional.

Abbreviations: FTND, Fagerstrom test for nicotine dependence; NDSS, nicotine dependence syndrome scale; NFO, National Family Opinion, Inc; NHIS, National Health Interview Survey; NRT, nicotine replacement therapy; OTC, over the counter; TTFC, time to first cigarette 
Also, NRT use increased when it was made available OTC, ,-6 $^{4-2}$ and may have attracted new users who had not availed themselves of NRT when it was prescription only. Finally, understanding how smokers self-select to use NRT is crucial for interpreting observed outcomes in self selected NRT users and non-users. ${ }^{511}$

We compared users and non-users of NRT on demographic and smoking characteristics. We particularly analysed how NRT use was related to a number of characteristics that might otherwise predispose smokers to failure in cessation. For example, heavier smokers are less likely to quit successfully: smoking more than 15 cigarettes per day is associated with a $40-50 \%$ decrease in the likelihood of quitting over a five year period. ${ }^{14}{ }^{15}$ Independent of smoking rates, those with greater nicotine dependence, as measured by Fagerstrom Tolerance Questionnaire scores, have one to two thirds less likelihood of success at quitting. ${ }^{16}{ }^{17}$ The experience of craving and withdrawal is also associated with failure; those with post-cessation craving scores in the highest quartile were eight times more likely to resume smoking, compared to those in the lowest quartile. ${ }^{18}$ Smokers with higher withdrawal scores were also more likely to fail. ${ }^{19}$ Duration of previous abstinence has also been shown to be associated with quitting success. For example, smokers who could attain abstinence exceeding 90 days in their previous quit had $80 \%$ better odds of remaining abstinent in a subsequent attempt. ${ }^{17}$ Confidence in one's ability to become a non-smoker has also been shown to be a highly significant predictor of abstinence. For example, before treatment onset, abstainers had higher expectations that they would stop for one day by the end of the course, were more likely to think they would quit permanently, and had higher self efficacy than early relapsers. ${ }^{20}$

Other, more distal factors are also associated with cessation success or failure. In one study, those with a history of smoking related medical illness were $25 \%$ less likely to remain quit, and relapsers had a 50\% higher rate of smoking related health problems than those who sustained abstinence. $^{21}$ It has been suggested that such diseases may represent markers of inability to quit, as many smokers quit shortly after the first appearance of smoking related symptoms, and those who persist in smoking despite the emergence of disease and consequent motivation and advice to quit constitute harder cases. ${ }^{21}$ Psychiatric morbidity has also been associated with failure in smoking cessation. Quit rates are lower among smokers who suffered mental illness (ranging from simple phobia to schizophrenia) in the past month $(30.5 \%)$ and in smokers with any lifetime history of mental illness $(37.1 \%)$ compared with smokers without mental illness $(42.5 \%)$. $^{22}$

Accordingly, we compared NRT users and non-users on a variety of factors associated with smoking cessation success. We also specifically examined those who used nicotine patch and gum since they became available OTC; this is because these smokers were able to self-select into NRT use without guidance from a physician, and because Pierce and Gilpin's ${ }^{6}$ comparison of self-selected OTC NRT users and non-users was thought to indicate lack of NRT efficacy.

\section{METHODS \\ Subjects}

Study participants were 9630 current adult smokers, aged 18 years and over, from households that were part of a national research panel maintained by National Family Opinion, Inc (NFO) and completed a mail survey regarding their current smoking and smoking history. The analysis was based on 7161 smokers who had ever tried to quit smoking.

We compared the obtained sample of respondents to the population characteristics of current smokers, as characterised in the 1999 National Health Interview Survey (NHIS). The analysis suggested that the sample of 9630 respondents were older and more likely to be female: $63 \%$ of current smokers in this sample were women, compared to $48 \%$ based on NHIS data, and $31 \%$ of current smokers in this sample were over 55 years of age, compared to only 16\% in the NHIS. The data were therefore weighted to match the age and sex distribution of US daily smokers in the 1999 NHIS. ${ }^{23}$ After this weighting, our study sample still overrepresented heavier, longer term smokers than the US population of smokers. For example, 92\% of our sample reported daily smoking, compared to only $82 \%$ in NHIS, and individuals smoking more than 20 cigarettes per day comprised $27 \%$ of our sample versus $16 \%$ nationally. Furthermore, $30 \%$ of our sample reported smoking for longer than 30 years, compared to only $16 \%$ in NHIS.

\section{Recruitment}

Households were initially recruited into the NFO panel via a mail invitation sent to households drawn from multiple marketing lists. Household and individual demographic information (including smoking status, based on a response to the question "Do you or does anyone in your household currently smoke?") was collected at entry into the panel and updated at least every two years (on a rotating basis). Households remain on the panel for an average of four years and are contacted about 10 times per year.

Our sample was drawn from a panel of 250000 households selected (from a larger NFO panel of nearly 575000 households) to be demographically representative of the noninstitutionalised population within the contiguous USA, according to the 2000 Current Population Survey from the US Census Bureau. ${ }^{24}$ Within each of nine geographic regions, the sub-panel is composed to match population data with regard to size of the metropolitan area, age (household head), income, and size of household. Analyses confirmed that the panel closely matched the US population profile (as reflected in the US Census) in each region. In selecting the sampling frame for this study, recently surveyed households (that is, those surveyed within the past 13 weeks-22.0\%) or those already being surveyed regarding smoking (5.5\%) were excluded in order to reduce respondent burden and possible bias from other surveys, and priority was given to households whose smoking status was most recently updated. A sampling frame of 16000 eligible households was selected to mirror the demographic profile of the USA.

\section{Procedure}

In February 2001, surveys and postage paid return envelopes were mailed to the 16000 households. A cover letter accompanying the survey directed the questionnaire to the member of the household who currently smoked cigarettes and was at least 18 years of age. If there was more than one qualifying smoker in the household, the respondent was to be selected quasi-randomly by targeting the smoker with the closest upcoming birthday. Respondents were compensated by an offer of $\$ 2$; typically, respondents are not compensated for participation in individual NFO surveys, except when surveys are lengthy, as this one was. Only one mailing was done, and receipt of responses was cut off after four weeks. Of the 16000 surveys that were mailed, 10061 were received within the allotted time, a response rate of $63 \%$. Though data are not available for non-respondents, the relatively high response rate should minimise response bias.

\section{Survey}

The eight page survey solicited detailed information about current smoking, smoking history, and quitting history. Smokers who reported having made at least one quit attempt 
were presented with a list of aids or programmes for quitting smoking (acupuncture, hypnosis, nicotine gum, nicotine patch, nicotine nasal spray, nicotine inhaler, prescription pill, stop smoking seminar/support group, self help materials, other) and were asked to designate which aids or programmes they had ever used. Respondents were also asked about their most recent attempt to quit, specifically when it occurred (classified as within six months, within a year, within three years, and more than three years ago) and which aids or programmes were used.

Respondents completed the Fagerstrom Test for Nicotine Dependence (FTND), ${ }^{25}$ a well validated measure of nicotine dependence, and the nicotine dependence syndrome scale (NDSS), a multifactor assessment of nicotine dependence that has demonstrated incremental validity over and above the FTND. ${ }^{26}$ Smokers were asked about the severity of craving and of several withdrawal symptoms (difficulty concentrating; irritability, frustration, or anger; insomnia or difficulty sleeping; depressed mood; restlessness; anxiety; increased appetite or weight gain) during their most recent quit effort. The rated intensity of individual withdrawal symptoms was averaged to obtain a composite rating of withdrawal severity. Respondents were also asked how difficult they thought it would be to quit smoking (0-10 scale) under scenarios involving assisted quitting (that is, quitting with gum or patch, gradually or abruptly) and unassisted quitting (that is, quitting without gum or patch, gradually or abruptly). These ratings were used to form indices of perceived difficulty with NRT assisted and unassisted quitting.

Finally, participants were asked to report whether they had personally experienced heart disease or circulatory conditions of any kind; lung disease or other respiratory conditions; and cancer other than lung. These were grouped into a single indicator of smoking related health problems. Participants were also asked to report whether they suffered anxiety/ nervousness; attention deficit disorder/hyperactivity; addictive behaviour besides smoking; or depression, and whether they were "overly stressed". These were also aggregated into a single index of psychiatric conditions.

\section{NRT user groups}

From the 10061 received questionnaires, 431 respondents were excluded because they reported not being 18 or older $(\mathrm{n}=54)$ or reported not being a current smoker $(\mathrm{n}=377)$. Since our analysis focused on methods of quitting smoking (rather than whether an attempt was made), we only included respondents who had made a quit attempt (2469 who had not made a quit attempt were excluded). Since our primary interest was in NRT use, we also excluded those respondents who reported having used prescription pills only ( $\mathrm{n}=322$; including these respondents made no substantial difference). The remaining 6839 respondents were classified according to their reported use of NRT: 2847 had ever used NRT (patch, gum, spray, inhaler) and 3992 were never-users. We next identified OTC NRT users by identifying those who said they had used nicotine patch or gum on their most recent quit attempt, and that this most recent quit attempt had taken place in the three years preceding the survey (1998-2001), which corresponded roughly to the OTC era (gum and patch became available OTC in late 1996). From among those who had ever used NRT, this yielded a sample of 979 OTC NRT users (dropping NRT users who did not report a quit attempt during the previous three years $(n=599)$ and those who had used a product other than gum or patch on their last quit attempt $(n=1269))$. A parallel group of 2469 non-users of NRT who had made a non-NRT quit attempt during the OTC era was constructed (that is, excluding those who had not made a quit attempt in three years $(\mathrm{n}=1523))$.

\section{Analysis}

We conducted three parallel sets of analyses: one compared NRT ever-users and never-users; another compared OTC NRT users and non-users (defined above); the third followed from the work of Pierce and Gilpin, ${ }^{6}$ who reported retrospective survey data showing no differences in abstinence duration among light smokers who used or did not use OTC NRT. Accordingly, we did an analysis of light smokers who reported smoking 15 cigarettes or fewer per day*, while adjusting for smoking rate, age, sex, and education, consistent with Pierce and Gilpin's approach. (We were not able to adjust for ethnicity, as this information was not collected in the survey.)

To assess whether the differences between NRT users and non-users could completely be accounted for by demographics or by crude indicators of dependence, we present analyses adjusted for age, sex, education, smoking rate, and time to first cigarette (TTFC; whether smokers smoked their first cigarette of the day within 30 minutes), a common indicator of dependence ${ }^{8}$ that is often included in epidemiological surveys. ${ }^{27}$ Pierce and Gilpin's ${ }^{6}$ analysis had adjusted for demographics and smoking rate, but not for TTFC.

We constructed strata for the predictor variables based on sample distributions and apparent breakpoints in the distributions. Logistic regression analyses estimated the odds ratios and their 95\% confidence intervals (OR, 95\% CI) comparing each stratum to the reference stratum, which was always the one associated with the greatest likelihood of success in quitting. We performed unadjusted and adjusted analyses (as described) of smoking history, tobacco dependence, quitting difficulty, and medical and psychiatric history. All statistical tests were considered significant at a two sided $\alpha$ level of $p \leqslant 0.05$. Data analyses were performed using SAS version 8.2 for Windows.

\section{RESULTS}

\section{Demographic characteristics}

We compared NRT ever-users and never-users. In unadjusted univariate analyses (table 1), men were more likely than women to have ever used NRT. NRT ever-users were also older and better educated, with college educated smokers having $40 \%$ higher odds of selecting NRT. Smokers with higher household income were more likely to have used NRT, significantly so when the annual household income exceeded $\$ 40$ 000. Similar differences were seen for OTC NRT users and non-users, with a trend towards stronger effects in the OTC comparisons.

\section{Smoking rate and time to first cigarette}

NRT ever-users were more likely to be heavy smokers: the odds of smoking over 20 cigarettes per day were doubled among the NRT users. The effects were even stronger for cessation efforts in the OTC era. The odds of being a heavy smoker were at least three times greater among OTC NRT users. NRT users also reported smoking their first cigarette earlier in the day, an index of nicotine dependence. NRT everusers were 2.9 times more likely to smoke within five minutes; for OTC users, this ratio rose to 4.7 times more likely.

\section{Smoking history}

The odds of being a daily smoker were greater among those who elected NRT use. Smokers who used NRT had been smoking longer than those who did not use NRT. The odds of NRT use were roughly doubled for those who had been

*Pierce and Gilpin defined light smokers as $<15$ cigarettes per day, but this cutoff yielded a small sample size, and the cutoff of $\leqslant 15$ cigarettes per day was deemed suitable 


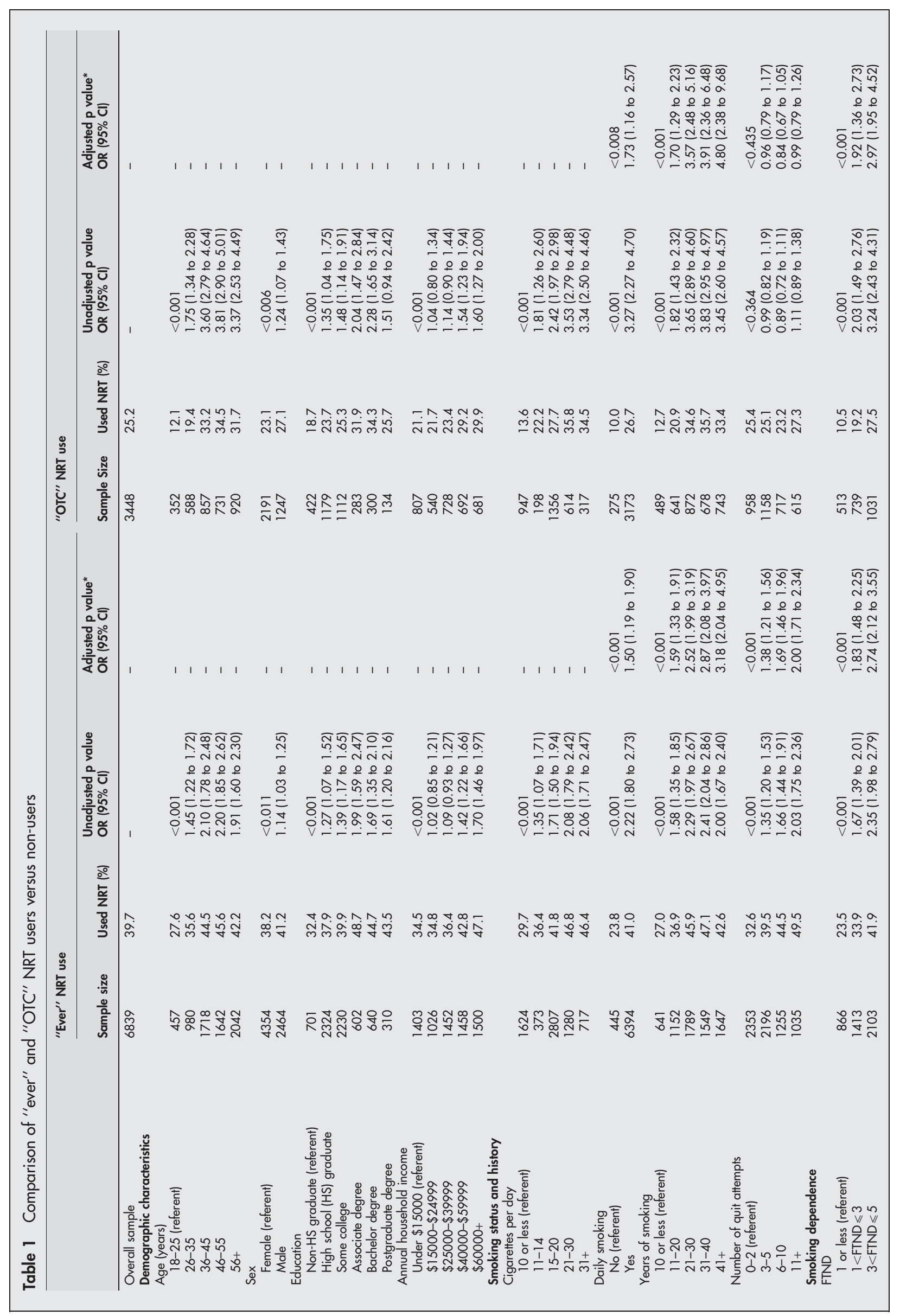




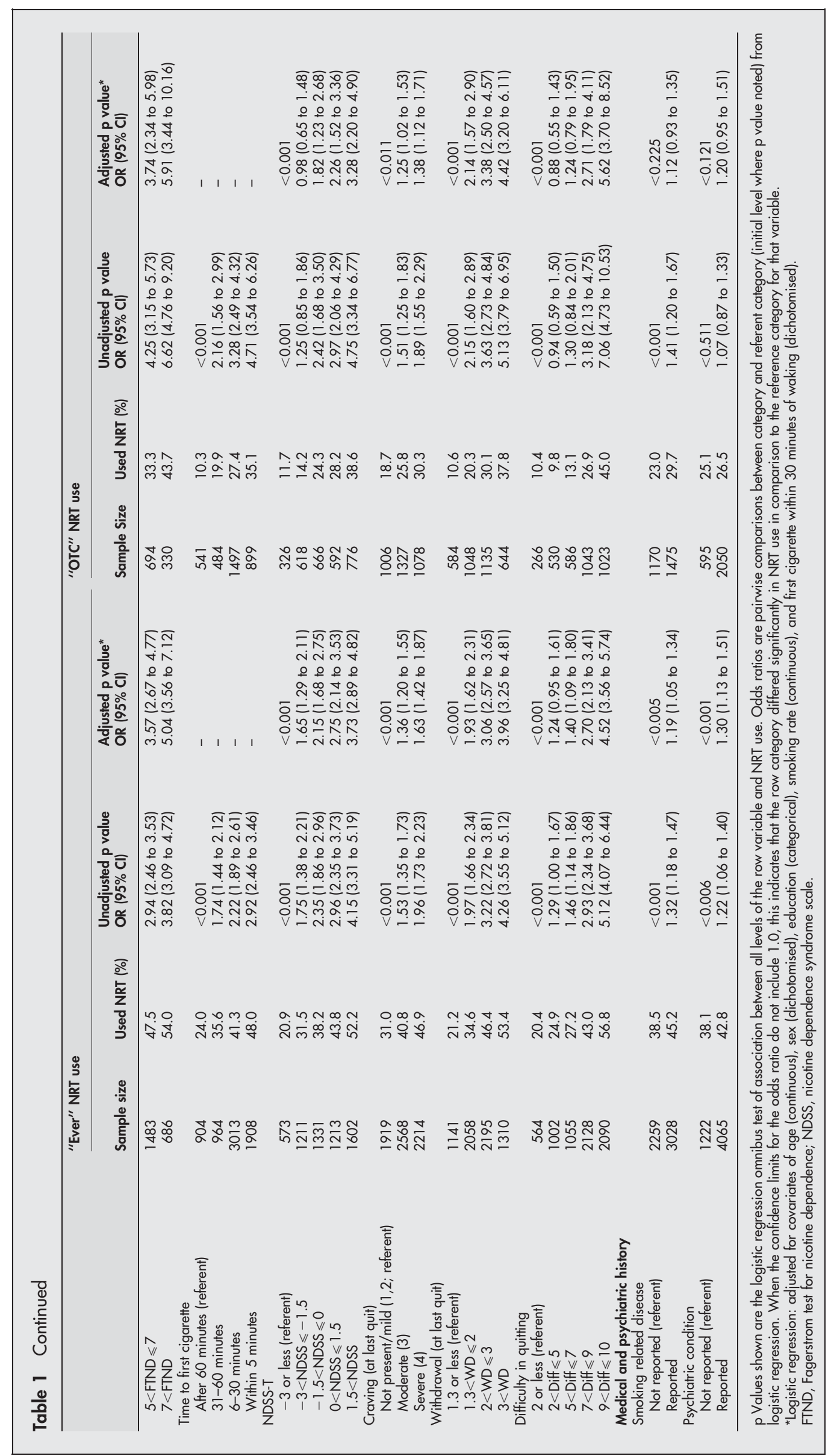


smoking for over 20 years. These effects were greater for OTC NRT users versus non-users. NRT ever-use was also more likely to occur among those who had made multiple quit attempts. This effect was not apparent among OTC NRT users, but the analysis is confounded because smokers who had not made a quit attempt within the last three years were excluded from analysis. All of these effects persisted even when the analysis adjusted for demographics, smoking rate, and TTFC.

\section{Tobacco dependence}

Those who selected NRT demonstrated greater tobacco dependence on the FTND measure of dependence. At the highest levels of FTND, the odds of NRT ever-use were more than three times higher than they were at the lowest stratum. The difference in FTND scores actually grew greater when we adjusted for smoking rate and TTFC. Contrasts of OTC NRT users and non-users revealed similar patterns, with smokers at the highest stratum being six times more likely to selfselect OTC NRT. These effects persisted at a reduced level even when adjusting for smoking rate and TTFC.

Similar findings were obtained for the NDSS, with the most dependent smokers being at least four times more likely to use NRT or OTC NRT. These differences persisted, at a reduced level, when smoking rate and TTFC were adjusted.

Smokers had been asked how much craving and withdrawal they experienced when they last quit smoking. NRT ever-users reported more intense craving and withdrawal. The odds of NRT use were over four times higher among those who reported the highest level of withdrawal symptoms. The same trends held for OTC NRT users, who showed a fivefold increase. (For this group, the most recent quit effort, which was the basis for symptom ratings, was the index quit effort on which they used OTC NRT.) Craving intensity also differentiated NRT users and OTC NRT users from non-users. These effects persisted in the analysis adjusted for smoking rate and TTFC.

\section{Medical and psychiatric history}

Those who had ever used NRT were more likely to report having experienced diseases associated with smoking (heart disease, lung disease, or cancer). Smokers with self reported psychiatric difficulties were also more likely to have ever used NRT. These effects persisted in the analysis adjusting for smoking rate and TTFC. Similar trends were evident for OTC NRT users, but the differences in psychiatric history were not significant, and medical history was not significant in the adjusted analysis.

\section{Expected difficulty quitting}

Smokers who expected difficulty quitting were much more likely to select NRT. The odds of using NRT were more than five times greater for the highest "difficulty" group as for the lowest; this effect was even stronger (OR $>7$ ) for OTC NRT users versus non-users. These differences persisted, at a reduced magnitude, when we adjusted for smoking rate and TTFC.

Because respondents had separately estimated the difficulty quitting with and without NRT treatment, we could assess these expectations without treatment and the expected benefit of NRT treatment. A repeated measures analysis of variance (ANOVA) examined the two difficulty quitting ratings for the two groups. There were significant differences between NRT ever-users and never-users $(\mathrm{F}(1,6831)=$ 462.34; $\mathrm{p}<0.001)$, and between ratings of NRT assisted and non-NRT assisted quit efforts $(F(1,6831)=1563.79$; $\mathrm{p}<0.001)$, but no interaction $(\mathrm{F}(\mathrm{l}, 6831)=3.26$; $\mathrm{p}<0.072$ ). As shown in fig 1 , both groups expected that they would find it easier to quit with NRT than without NRT (as evidenced by the similar gap between adjacent bars in fig 1), but NRT users expected greater difficulty quitting under both conditions (as evidenced by the higher bars for this group in fig 1). Indeed, the degree of difficulty quitting anticipated by NRT users was so much that the beneficial effect expected of NRT only brought their difficulty down to the level expected by the non-users for unaided quitting. Similar patterns emerged in analyses of OTC NRT users and non-users: there were significant differences between OTC NRT users and non-users $(F(1,3441)=265.86$; $p<0.001)$ and between ratings of NRT assisted and non-NRT assisted quit efforts $(\mathrm{F}(1,3441)=813.25 ; \mathrm{p}<0.001)$, although the presence of a significant interaction $(F(1,3441)=6.27$; $\mathrm{p}<0.013$ ) showed that OTC NRT users expected even more difficulty with an unaided quitting method. Again, OTC NRT users expected NRT simply to "normalize" their difficulty quitting to the level of non-NRT users.

\section{OTC NRT use among light smokers}

Relevant to Pierce and Gilpin's ${ }^{6}$ analysis of quitting outcomes among light smokers who used or did not use OTC NRT, we also examined OTC era NRT use among light smokers $(\leqslant 15$ cigarettes/day) (table 2). In this analysis, as in the prior one, OTC NRT users tended to be older, male, and relatively heavier smokers. In subsequent analyses, following Pierce and Gilpin's published procedure, we controlled for age, sex, education, and smoking rate. Nevertheless, the results in this narrower group of light smokers largely mirrored the trends

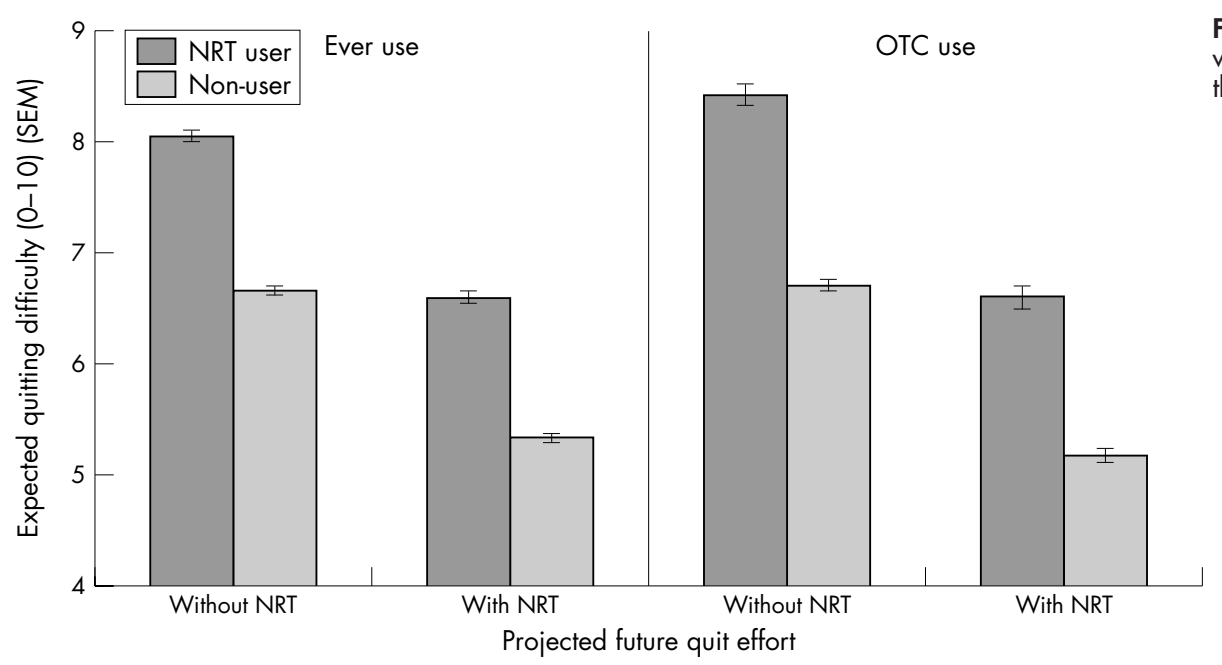

Figure 1 Expected difficulty quitting, with and without nicotine replacement therapy (NRT). 
Table 2 Comparison of "OTC" NRT users versus non-users among lighter smokers*

\begin{tabular}{|c|c|c|c|c|c|}
\hline & \multicolumn{5}{|c|}{ Smokers $\leqslant 15$ cigarettes/day: "OTC" NRT use } \\
\hline & Sample size & Used NRT (\%) & $\begin{array}{l}\text { Unadiusted } \mathrm{p} \text { value } \\
\text { OR }(95 \% \mathrm{CI})\end{array}$ & $\begin{array}{l}\text { Adjusted } p \text { valuet } \\
\text { OR }(95 \% \mathrm{CI})\end{array}$ & $\begin{array}{l}\text { Adjusted } \mathrm{p} \text { valuef } \\
\text { OR }(95 \% \mathrm{CI})\end{array}$ \\
\hline $\begin{array}{l}\text { Overall sample } \\
\text { Demographic characteristics }\end{array}$ & 1554 & $16.4 \%$ & - & - & - \\
\hline \multicolumn{6}{|l|}{$\begin{array}{l}\text { Demographic characteristics } \\
\text { Age (years) }\end{array}$} \\
\hline $18-25$ (referent) & 208 & 9.7 & $<0.001$ & - & - \\
\hline $26-35$ & 318 & 12.0 & $1.26(0.84$ to 1.90$)$ & - & - \\
\hline $36-45$ & 370 & 22.5 & $2.69(1.82$ to 3.99$)$ & - & - \\
\hline $46-55$ & 263 & 25.5 & 3.18 (2.05 to 4.93$)$ & - & - \\
\hline $56+$ & 395 & 21.8 & 2.58 (1.64 to 4.07$)$ & - & - \\
\hline \multicolumn{6}{|l|}{ Sex } \\
\hline Female (referent) & 1072 & 15.3 & $<0.167$ & - & - \\
\hline Male & 476 & 17.8 & $1.20(0.93$ to 1.54$)$ & - & - \\
\hline \multicolumn{6}{|l|}{ Education } \\
\hline Non-HS graduate (referent) & 177 & 16.9 & $<0.315$ & - & - \\
\hline High school (HS) graduate & 479 & 16.4 & $0.96(0.63$ to 1.47$)$ & - & - \\
\hline Some college & 511 & 14.4 & $0.83(0.54$ to 1.26$)$ & - & - \\
\hline Associate degree & 143 & 18.6 & $1.12(0.65$ to 1.93$)$ & - & - \\
\hline Bachelor degree & 160 & 21.6 & $1.36(0.81$ to 2.26$)$ & - & - \\
\hline Postgraduate degree & 75 & 13.7 & $0.78(0.36$ to 1.68$)$ & - & - \\
\hline \multicolumn{6}{|l|}{ Annual household income } \\
\hline Under \$15000 (referent) & 357 & 14.0 & $<0.275$ & - & - \\
\hline$\$ 15000-\$ 24999$ & 234 & 15.7 & $1.15(0.74$ to 1.78$)$ & - & - \\
\hline$\$ 25000-\$ 39999$ & 310 & 15.2 & $1.10(0.73$ to 1.65$)$ & - & - \\
\hline$\$ 40000-\$ 59999$ & 328 & 17.0 & $1.26(0.85$ to 1.87$)$ & - & - \\
\hline$\$ 60000+$ & 325 & 19.7 & 1.50 (1.02 to 2.21$)$ & - & - \\
\hline \multicolumn{6}{|l|}{ Smoking status and history } \\
\hline \multicolumn{6}{|l|}{ Cigarettes per day } \\
\hline 10 or less (referent) & 947 & 13.6 & $<0.001$ & - & - \\
\hline $11-14$ & 198 & 22.2 & $1.81(1.26$ to 2.60$)$ & - & - \\
\hline $15-20$ & 409 & 20.5 & $1.63(1.22$ to 2.18$)$ & - & - \\
\hline $21-30$ & - & - & - & - & - \\
\hline $31+$ & - & - & - & - & - \\
\hline Daily smoking & & & & & \\
\hline No (referent) & 257 & 10.4 & $<0.002$ & $<0.395$ & $<0.616$ \\
\hline Yes & 1297 & 17.8 & $1.87(1.27$ to 2.74$)$ & $1.21(0.78$ to 1.87$)$ & $1.12(0.72$ to 1.76$)$ \\
\hline Years of smoking & & & & & \\
\hline 10 or less (referent) & 312 & 10.6 & $<0.001$ & $<0.001$ & $<0.001$ \\
\hline $11-20$ & 343 & 12.2 & $1.17(0.80$ to 1.71$)$ & $1.16(0.75$ to 1.79$)$ & $1.13(0.73$ to 1.74$)$ \\
\hline $21-30$ & 341 & 25.1 & $2.82(1.98$ to 4.04$)$ & 3.00 (1.68 to 5.34$)$ & $2.90(1.63$ to 5.17$)$ \\
\hline $31-40$ & 240 & 25.5 & 2.88 (1.88 to 4.43$)$ & 3.09 (1.37 to 6.95$)$ & 2.80 (1.24 to 6.33 ) \\
\hline $41+$ & 306 & 23.2 & 2.55 (1.62 to 4.00$)$ & $3.26(1.03$ to 10.38$)$ & $3.01(0.95$ to 9.59$)$ \\
\hline Number of quit attempts & & & & & \\
\hline $0-2$ (referent) & 443 & 17.6 & $<0.453$ & $<0.553$ & $<0.480$ \\
\hline $3-5$ & 519 & 14.5 & 0.79 (0.58 to 1.09$)$ & $0.79(0.57$ to 1.10$)$ & 0.77 (0.55 to 1.07$)$ \\
\hline $6-10$ & 332 & 16.8 & 0.95 (0.67 to 1.35 ) & $0.92(0.64$ to 1.32$)$ & 0.91 (0.63 to 1.31$)$ \\
\hline $11+$ & 260 & 17.9 & $1.02(0.69$ to 1.51$)$ & $0.92(0.62$ to 1.37$)$ & 0.90 (0.60 to 1.35$)$ \\
\hline Smoking dependence & & & & & \\
\hline FTND & & & & & \\
\hline 1 or less (referent) & 481 & 10.8 & $<0.001$ & $<0.001$ & $<0.015$ \\
\hline $1<$ FTND $\leqslant 3$ & 507 & 15.3 & $1.49(1.06$ to 2.11$)$ & $1.23(0.84$ to 1.80$)$ & $1.26(0.81$ to 1.96$)$ \\
\hline $3<$ FTND $\leqslant 5$ & 394 & 22.4 & $2.38(1.69$ to 3.37$)$ & $1.78(1.17$ to 2.71$)$ & $1.86(1.02$ to 3.41$)$ \\
\hline $5<$ FTND $\leqslant 7$ & 97 & 32.7 & $4.01(2.45$ to 6.57$)$ & $2.92(1.65$ to 5.17$)$ & $3.06(1.46$ to 6.40$)$ \\
\hline $7<$ FTND & - & - & - & - & - \\
\hline Time to first cigarette & & & & & \\
\hline Affer 60 minutes (referent) & 474 & 10.6 & $<0.001$ & $<0.007$ & - \\
\hline $31-60$ minutes & 321 & 15.7 & $1.57(1.06$ to 2.31$)$ & $1.30(0.87$ to 1.95$)$ & - \\
\hline 6-30 minutes & 595 & 19.6 & $2.06(1.48$ to 2.85$)$ & $1.53(1.06$ to 2.20$)$ & - \\
\hline Within 5 minutes & 149 & 27.3 & $3.16(2.03$ to 4.90$)$ & $2.32(1.43$ to 3.75$)$ & - \\
\hline NDSS-T & & & & & \\
\hline-3 or less (referent) & 276 & 11.2 & $<0.001$ & $<0.001$ & $<0.003$ \\
\hline$-3<$ NDSS $\leqslant-1.5$ & 416 & 12.5 & $1.13(0.72$ to 1.77$)$ & $0.88(0.55$ to 1.42$)$ & $0.84(0.52$ to 1.36$)$ \\
\hline$-1.5<$ NDSS $\leqslant 0$ & 301 & 18.9 & $1.84(1.18$ to 2.86$)$ & 1.51 (0.94 to 2.41$)$ & $1.43(0.89$ to 2.30$)$ \\
\hline $0<$ NDSS $\leqslant 1.5$ & 202 & 19.8 & $1.95(1.22$ to 3.13$)$ & $1.64(0.98$ to 2.75$)$ & $1.52(0.91$ to 2.57$)$ \\
\hline $1.5<$ NDSS & 135 & 28.7 & $3.19(1.95$ to 5.20$)$ & 2.47 (1.44 to 4.23$)$ & 2.14 (1.23 to 3.73$)$ \\
\hline Craving (at last quit) & & & & & \\
\hline Not present/mild (1,2; referent) & 609 & 12.9 & $<0.002$ & $<0.044$ & $<0.115$ \\
\hline Moderate (3) & 634 & 16.7 & $1.34(1.00$ to 1.81$)$ & 1.11 (0.82 to 1.52$)$ & $1.10(0.81$ to 1.50$)$ \\
\hline Severe (4) & 291 & 21.9 & $1.89(1.34$ to 2.65$)$ & 1.56 (1.09 to 2.24$)$ & $1.46(1.01$ to 2.10$)$ \\
\hline Withdrawal (at last quit) & & & & & \\
\hline 1.3 or less (referent) & 356 & 7.8 & $<0.001$ & $<0.001$ & $<0.001$ \\
\hline $1.3<W D \leqslant 2$ & 563 & 14.8 & 2.04 (1.33 to 3.12 ) & $1.87(1.21$ to 2.90$)$ & 1.78 (1.15 to 2.77$)$ \\
\hline $2<W D \leqslant 3$ & 443 & 21.7 & $3.26(2.13$ to 5.00$)$ & $3.07(1.98$ to 4.76$)$ & 2.88 (1.85 to 4.50$)$ \\
\hline $3<W D$ & 172 & 26.7 & $4.29(2.61$ to 7.06$)$ & 3.71 (2.21 to 6.22$)$ & 3.43 (2.03 to 5.78$)$ \\
\hline Difficulty in quitting & & & & & \\
\hline 2 or less (referent) & 162 & 4.6 & $<0.001$ & $<0.001$ & $<0.001$ \\
\hline $2<$ Diff $\leqslant 5$ & 325 & 8.3 & $1.85(0.85$ to 4.01$)$ & 1.56 (0.71 to 3.42$)$ & $1.48(0.67$ to 3.25$)$ \\
\hline $5<$ Diff $\leqslant 7$ & 341 & 9.2 & 2.08 (0.97 to 4.43$)$ & 1.82 (0.85 to 3.93$)$ & 1.79 (0.83 to 3.85$)$ \\
\hline $7<$ Diff $\leqslant 9$ & 430 & 21.5 & 5.61 (2.74 to 11.49$)$ & $4.58(2.21$ to 9.50$)$ & $4.41(2.13$ to 9.16$)$ \\
\hline $9<$ Diff $\leqslant 10$ & 296 & 36.5 & 11.81 (5.73 to 24.32 ) & 9.24 (4.42 to 19.32$)$ & $8.72(4.16$ to 18.29$)$ \\
\hline
\end{tabular}


Table 2 Continued

\begin{tabular}{|c|c|c|c|c|c|}
\hline & \multicolumn{5}{|c|}{ Smokers $\leqslant 15$ cigarettes/day: "OTC" NRT use } \\
\hline & Sample size & Used NRT (\%) & $\begin{array}{l}\text { Unadjusted } p \text { value } \\
\text { OR }(95 \% \mathrm{CI})\end{array}$ & $\begin{array}{l}\text { Adjusted } p \text { valuet } \\
\text { OR }(95 \% \mathrm{Cl})\end{array}$ & $\begin{array}{l}\text { Adjusted } p \text { valueł } \\
\text { OR }(95 \% \mathrm{CI})\end{array}$ \\
\hline \multicolumn{6}{|c|}{ Medical and psychiatric history } \\
\hline \multicolumn{6}{|c|}{ Smoking related disease } \\
\hline Not reported (referent) & 542 & 15.5 & $<0.007$ & $<0.170$ & $<0.139$ \\
\hline Reported & 600 & 21.4 & 1.49 (1.12 to 1.98$)$ & 1.25 (0.91 to 1.71$)$ & $1.27(0.93$ to 1.74$)$ \\
\hline \multicolumn{6}{|l|}{ Psychiatric condition } \\
\hline Not reported (referent) & 296 & 19.5 & $<0.475$ & $<0.756$ & $<0.526$ \\
\hline Reported & 846 & 17.7 & $0.89(0.63$ to 1.24$)$ & 0.94 (0.66 to 1.34$)$ & 0.89 (0.63 to 1.27$)$ \\
\hline \multicolumn{6}{|c|}{$\begin{array}{l}\text { p Values shown are the logistic regression omnibus test of association between all levels of the row variable and NRT use. Odds ratios are pair wise comparisons } \\
\text { between category and referent category (initial level where p value noted) from logistic regression. When the confidence limits for the odds ratio do not include } \\
1.0 \text {, this indicates that the row category differed significantly in NRT use in comparison to the reference category for that variable. } \\
\text { *This subanalysis was performed to be consistent with Pierce and Gilpin's analysis of quitting outcomes among light smokers who used or did not use OTC NRT. } \\
\text { †Logistic regression: adjusted for covariates of age (continuous), sex (dichotomised), education (categorical), and smoking rate (continuous). } \\
\text { flogistic regression: adjusted for covariates of age (continuous), sex (dichotomised), education (categorical), smoking rate (continuous), and first cigarette within } \\
30 \text { minutes of waking (dichotomised). }\end{array}$} \\
\hline
\end{tabular}

seen in the main analyses that considered all OTC users and non-users. Compared to non-users, light smoker OTC NRT users had been smoking longer. They also showed more severe nicotine dependence, both on the FTND and on the NDSS measures; on both measures, it was particularly likely that OTC NRT users would be in the highest stratum of nicotine dependence. Light smokers who elected to use OTC NRT reported more intense craving, more intense nicotine withdrawal, and expected more difficulty quitting at their last quit attempt. Indeed, smokers in the group that expected the greatest difficulty quitting had in excess of nine times greater odds of being NRT users. Light smoking OTC NRT users were not distinguished by medical and psychiatric history. Extending Pierce and Gilpin's report, we additionally adjusted the analysis for TTFC. This only slightly attenuated the differences between OTC NRT users and non-users, and all of the significant effects persisted even with adjustment for TTFC.

\section{DISCUSSION}

This analysis gives some insight into the characteristics of NRT users and shows that smokers who have elected to use NRT and/or been prescribed NRT differ in numerous ways from those who have not. The pattern of differences generally suggested that NRT users would have had more difficulty quitting successfully: they were longer term smokers, showed more severe nicotine dependence, had more intense craving and withdrawal when quitting, and expected greater difficulty quitting. This suggests that NRT medications are being used by those at greatest risk for failure and also has methodological implications for population studies of NRT.

On every index of dependence we examined, the smokers who elected NRT use were more dependent: they were heavier smokers, had smoked for a longer time, had experienced more intense craving and withdrawal upon quitting, and scored higher on the FTND and NDSS measures of dependence severity. This was true for smokers who had used NRT during the US OTC era, when smokers could obtain NRT without a prescription, as well as for ever-users of NRT, who may have been prescribed NRT by a physician. This suggests that both smokers and prescribers made considered choices in electing to use NRT, providing for NRT use in those who were at greatest risk for cessation failure and who needed it most. Many studies indicate that NRT use is most beneficial to the most dependent smokers, because more dependent smokers fare particularly poorly when they try to quit without NRT treatment; NRT reverses this excess risk of failure and allows highly dependent smokers to achieve outcomes comparable to those of less dependent smokers. ${ }^{101128}$

NRT use was also more common among smokers who harboured other risk factors for smoking cessation failure. Ever-users of NRT were more likely to have diseases that could be smoking related. Existing disease often marks a smoker who is refractory to quitting and has continued to smoke despite pressure and incentives to quit; smokers who already suffer from disease are generally less able to quit. ${ }^{17} 29$ NRT users also reported suffering from psychiatric comorbidities, which are also associated with failure in smoking cessation. ${ }^{22}{ }^{30}$ Similar trends were observed among more recent users of OTC NRT, but the effects did not reach significance. Medical and psychiatric conditions may bring smokers into contact with medical providers, resulting in increased NRT use under a prescription system, but not under OTC conditions.

Given the risks of failure that are associated with selection of NRT, it is not surprising that smokers who had elected NRT use expected greater difficulty quitting-even with NRT. Indeed, their expected difficulty with NRT was roughly comparable to the difficulty that non-NRT users expected for quitting without treatment. It appears that these heavier, more dependent smokers who anticipate failing in smoking cessation look to NRT to at least "normalise" their likelihood of success up to the level of the lighter, less dependent smokers who do not feel they need NRT treatment. This mirrors the pattern seen in actual success rates, where NRT "normalises" the success rate of highly dependent smokers, rendering them equal to those in less dependent smokers. ${ }^{101128}$

In summary, the findings indicate that NRT users and nonusers are different populations. NRT users had multiple risk factors for failure in smoking cessation, implying that, without NRT, they would have considerably poorer chances of quitting successfully. An important methodological implication is that the comparison of naturally occurring groups of self-selected NRT users and non-users for purposes of assessing the effectiveness of NRT is likely to be problematic, as the two groups "naturally" differ in the outcomes they can expect in smoking cessation, even before factoring in the effect of treatment. We tested whether the group differences we observed could easily be statistically adjusted by controlling for simple and often used indices of dependencesmoking rate and smoking within 30 minutes of waking. The analysis showed that these variables did not account for most of the differences, suggesting that, despite the inclusion of such items in many surveys (including the California Tobacco Survey ${ }^{27}$ ), they do not constitute an adequate control. This 
highlights the difficulty of knowing when one has adequately controlled all relevant group differences. While statistical adjustment for known covariates can be used to reduce bias, complete control of population differences is rarely feasible, as there are often additional important but unmeasured covariates. ${ }^{31}$ Thus, the failure of retrospective surveys to find higher success rates among NRT users does not imply that NRT is not effective. Pierce and Gilpin compared groups of self-selected NRT users and non-users, and found only modest superiority in outcomes for the NRT users. ${ }^{6}$ In a further analysis focusing specifically on light smokers, comparing outcomes achieved by OTC NRT users and nonusers, Pierce and Gilpin concluded that there were no differences in outcome, and that NRT was ineffective in light smokers. However, our analysis shows that these selfselected NRT users and non-users are not comparable. Even an analysis limited to light smokers, and further controlling for smoking rate, age, sex, and education (as Pierce and Gilpin had done in an attempt to control for self-selection) still demonstrated consistent and often dramatic differences between those who elected to use OTC NRT and those who did not. For example, those who used OTC NRT were more nicotine dependent, had experienced more severe nicotine withdrawal symptoms, and anticipated greater difficulty quitting. These differences persisted even after we controlled for TTFC, which Pierce and Gilpin had not done.

Thus, the finding that self-selected NRT users identified retrospectively by survey do not always achieve better outcomes than non-NRT users does not mean that NRT did not help them. Indeed, the fact that the high risk NRT selectors achieved outcomes comparable to those of the less dependent smokers who did not seek NRT may actually demonstrate the benefit of NRT. This is parallel to the pattern seen in smokers' expectancies of difficulty quitting (fig 1), which shows that NRT is expected to provide a clear benefit in quitting. However, since the smokers who elect to use NRT have greater difficulty quitting in the first place, the effect of this benefit is to bring their expected difficulty in line with that of the lighter, less dependent smokers quitting without NRT - that is, the difficulty of NRT users on NRT is equal to that of non-NRT users without NRT, but this comparison fails to capture the substantial benefit of NRT. Indeed, outcome analyses $^{10}$ show that this pattern carries over into quitting success: NRT undoes the disadvantage of nicotine dependence to bring highly dependent smokers' quit rates into line with those of less dependent ones. Because the decision to use NRT is itself based on risk for poor outcomes, comparison of outcomes between self-selected NRT users and non-users is uninformative and even misleading regarding treatment effects.

Such "indication bias" is a well known confound in population studies of treatment outcomes, ${ }^{32}{ }^{33}$ and makes retrospective comparison of groups who self-select NRT use, as in Pierce and Gilpin, ${ }^{6}$ not a useful way to assess treatment efficacy. Although there is concern that people who enrol in clinical trials are not representative, randomised clinical trials are considered the gold standard for evaluating treatment effects precisely because they eliminate indication bias associated with self-selection. Many randomised trials have found that NRT is effective, even under OTC conditions. ${ }^{34}$

Our data showed that NRT is less likely to be used by lighter and less dependent smokers. While self selection of NRT by the most highly dependent smokers is in some ways appropriate, the lower utilisation among less dependent smokers represents a missed opportunity. While the efficacy of NRT is relatively higher among more dependent smokers, NRT also helps less dependent smokers achieve success in quitting. $^{10}$ It was particularly striking to see that the gradient of NRT use by smoking rate has grown steeper in

\section{What this paper adds}

In randomised clinical trials, nicotine replacement therapy (NRT) has been shown to promote smoking cessation in a variety of smoking populations. NRT utilisation is increasing, and smokers can decide whether or not to use NRT without consulting a doctor. However, retrospective surveys comparing self selected users and non-users of OTC NRT sometimes fail to find robust differences in cessation rates, possibly because smokers who elect to use NRT start with greater risk for cessation failure.

This study showed that smokers who elect to use NRT differ from non-NRT users in a number of ways that predispose them to failure in cessation: they were longer term smokers, showed more severe nicotine dependence, had more intense craving and withdrawal when quitting, and expected greater difficulty quitting. As such, NRT is being used by those who need it most. However, these differences between NRT users and non-users make the groups non-comparable, biasing any outcome comparisons based on self selected groups.

the OTC era, with NRT use shifting even more towards heavier smokers. Smokers may need to be educated that NRT can help lighter smokers.

The differences observed between NRT users and nonusers were roughly similar whether one considered lifetime use or use during the OTC era, but were somewhat bigger in the OTC era. This suggests that self-selection for NRT use may be more influenced by dependence. Prescribers may have been more likely to offer NRT to a wider range of smokers, whereas smokers may attend more to their dependence and expected difficulty quitting in deciding to use NRT. The relatively similar findings for OTC NRT users and NRT everusers may be due to partial overlap between the two groups (with $34 \%$ of ever NRT users also classified as OTC NRT users). It is worth noting that evidence suggests that most prescriptions for NRT were initiated by request of the smoker, rather than the prescriber; ${ }^{35-37}$ in effect, use of NRT was selfselected even during the prescription era.

Demographically, NRT was more likely to be used by relatively educated smokers with higher incomes. These smokers may be better informed about the harm of smoking and more motivated to quit. They may also be better able to afford to pay for NRT medications, or more likely to have health coverage for treatment. Moreover, in contrast to most of the attributes that characterise NRT users, wealth and education are associated with success in quitting, ${ }^{14}{ }^{38}$ so these associations pull against the trends discussed above. It was surprising to see that men were more likely to use NRT than women, particularly as women are more likely than men to use help in their attempts to stop smoking; ${ }^{39}$ men may find the medical approach to quitting implicit in NRT treatment more palatable than behavioural approaches.

Like any study, ours had limitations that should be taken into account in interpreting the results. A survey of respondents from a standing research panel may not be representative of the US population, and non-response may have further skewed our survey sample. Even after weighting to match the age and sex distribution of current smokers in the USA, our study sample overrepresented heavier, longer term smokers than the US population of smokers. It is possible that heavier smokers may have been more likely to respond to a survey about smoking. In any case, the restriction of range indicated by these sampling effects probably reduced the apparent differences between NRT users and non-users, so our findings may be conservative. Another limitation of this study is that we only surveyed 
current smokers, and did not include ex-smokers. Thus, by definition, we only examined failed past quit efforts. This may have conservatively biased our analyses of dependence; since NRT is known to preferentially increase success for highly dependent smokers, ${ }^{10}$ the differences in dependence between NRT users and non-users may be even greater than what we observed. Another limitation is that our assessment of NRT use during the OTC era was incomplete; we only captured cases where NRT was used for the most recent quit attempt, and those that occurred starting 12-18 months after patch and gum became OTC. Finally, all of our data were based on self report, and may therefore be subject to problems associated with recall or other biases.

In summary, we found that NRT use is higher among smokers who are more nicotine dependent and have greater risk of failure in smoking cessation, which suggests that NRT is reaching the populations who need it most. Importantly, the finding that NRT users differ from non-users in myriad ways that predispose them to greater difficulty in smoking cessation implies that correlational comparisons of outcome between self-selected NRT users and non-users can be significantly biased, in ways that cannot readily be adjusted by statistical controls. This implies that great caution is called for in outcome comparisons between self-selected NRT users and non-users.

\section{ACKNOWLEDGEMENTS}

We wish to acknowledge Gary Giovino for his valuable input on an earlier draft of this manuscript.

\section{Authors' affiliations \\ S Shiffman, Pinney Associates and University of Pittsburgh, Pittsburgh, Pennsylvania, USA}

M E Di Marino, C T Sweeney, Pinney Associates, Bethesda, Maryland, USA

Financial disclosure: This study was supported by GlaxoSmithKline Consumer Healthcare (GSKCH), which markets nicotine replacement medications for smoking cessation. Drs Shiffman and Sweeney and $\mathrm{Mr}$ Di Marino serve as consultants to GSKCH on an exclusive basis in matters relating to smoking cessation. Dr Shiffman also has an interest in a venture to develop a new nicotine replacement product.

\section{REFERENCES}

Mokdad AH, Mark JS, Stroup DF, et al. Actual causes of death in the United States, 2000. JAMA 2004;291:1238-45.

2 Hughes JR, Keely J, Naud S. Shape of the relapse curve and long-term abstinence among untreated smokers. Addiction 2004;99:29-38.

3 Fiore MC, Bailey WC, Cohen SJ, et al. Treating tobacco use and dependence. Clinical Practice Guideline. Rockville, Maryland: US Department of Health and Human Services, Public Health Service, 2000.

4 Shiffman S, Gitchell J, Pinney JM, et al. Public health benefit of over-thecounter nicotine medications. Tobacco Control 1997;6:306-10.

5 Burton SL, Gitchell JG, Shiffman S. Use of FDA-approved pharmacologic treatments for tobacco dependence - United States, 1984-1998. MMWR Morb Mortal Wkly Rep 2000;49:665-8

6 Pierce JP, Gilpin EA. Impact of over-the-counter sales on effectiveness of pharmaceutical aids for smoking cessation. JAMA 2002;288:1260-4.

7 Cummings KM, Hyland A, Ockene JK, et al. Use of the nicotine skin patch by smokers in 20 communities in the United States, 1992-1993. Tobacco Control 1997;6:S63-70

8 Heatherton TF, Kozlowski LT, Frecker RC, et al. Measuring the heaviness of smoking: using self-reported time to the first cigarette of the day and number of cigarettes smoked per day. Addiction 1989;84:791-800.
9 Shiffman S. Nicotine lozenge efficacy in light smokers. Drug Alcohol Depend 2005;77:311-14.

10 Shiffman S, Paton SM. Individual differences in smoking: gender and nicotine addiction. Nicotine Tob Res 1999;1(suppl 2):S153-7.

11 Shiffman S, Dresler CM, Hajek P, et al. Efficacy of a nicotine lozenge for smoking cessation. Arch Intern Med 2002;162:1267-76.

12 Silagy C, Lancaster T, Stead L, et al. Nicotine replacement therapy for smoking cessation (Cochrane Review). In: The Cochrane Library, Issue 1. Chichester UK: John Wiley \& Sons, Ltd, 2004.

13 White $\mathbf{P}$, Watt J. Tobacco control in London: facts and issues. London: SomekeFree London, 2002.

14 Hymowitz N, Cummings KM, Hyland A, et al. Predictors of smoking cessation in a cohort of adult smokers followed for five years. Tobacco Control 1997;6:S57-62.

15 Nordstrom BL, Kinnunen T, Utman CH, et al. Predictors of continued smoking over 25 years of follow-up in the normative aging study. Am J Public Health 2000;90:404-6.

16 Pinto RP, Abrams DB, Monti PM, et al. Nicotine dependence and likelihood of quitting smoking. Addictive Behaviors 1987;12:371-4.

17 Hill HA, Schoenbach VJ, Kleinbaum DG, et al. A longitudinal analysis of predictors of quitting smoking among participants in a self-help intervention trial. Addictive Behaviors 1994; 19:159-73.

18 Killen JD, Fortmann SP. Craving is associated with smoking relapse: findings from three prospective studies. Exp Clin Psychopharmacol 1997:5:137-42.

19 West RJ, Hajek P, Belcher M. Severity of withdrawal symptoms as a predictor of outcome of an attempt to quit smoking. Psychol Med 1989;19:981-5.

20 Stuart K, Borland R, McMurray N. Self-efficacy, health locus of control, and smoking cessation. Addictive Behaviors 1994;19:1-12.

21 Monso E, Campbell J, Tonnesen P, et al. Sociodemographic predictors of success in smoking intervention. Tobacco Control 2001;10:165-9.

22 Lasser K, Boyd JW, Woolhandler S, et al. Smoking and mental illness: A population-based prevalence study. JAMA 2000;284:2606-10.

23 National Center for Health Statistics. Dataset documentation, National Health Interview Survey, 1999. Hyattsville, Maryland: National Center for Health Statistics, 2002.

24 US Department of Labor, US Department of Commerce. Current Population Survey. Technical Paper 63. Design and Methodology. http:// www.census.gov/prod/2000pubs/tp63.pdf, 2000.

25 Heatherton TF, Kozlowski LT, Frecker RC, et al. The Fagerstrom test for nicotine dependence: a revision of the Fagerstrom tolerance questionnaire. Br J Addiction 1991;86:1119-27.

26 Shiffman S, Waters A, Hickcox M. The nicotine dependence syndrome scale: a multidimensional measure of nicotine dependence. Nicotine Tob Res 2004;6:327-48.

27 Pierce JP, Cavin SW, Macky C, et al. Technical report on analytic methods and approaches used in the 1993 California tobacco survey analysis. Sacramento, California: California Department of Health Services, 1994.

28 Fagerstrom KO, Schneider NG. Measuring nicotine dependence: a review of the Fagerstrom tolerance questionnaire. J Behav Med 1989;12:159-82.

29 Matheny KB, Weatherman KE. Predictors of smoking cessation and maintenance. J Clin Psychol 1998;54:223-35.

30 Glassman AH, Helzer JE, Covey LS, et al. Smoking, smoking cessation, and major depression. JAMA 1990;264:1546-9.

31 Klungel OH, Martens EP, Psaty BM, et al. Methods to assess intended effects of drug treatment in observational studies are reviewed. J Clin Epidemiol 2004;57:1223-31.

32 Rockwood K, Kirkland S, Hogan DB, et al. Use of lipid-lowering agents, indication bias, and the risk of dementia in community-dwelling elderly people. Arch Neurol 2002;59:223-7.

33 Ness RB, Cauley JA. Antibiotics and breast cancer-what's the meaning of this? JAMA 2004:291:880-1.

34 Hughes JR, Shiffman S, Callas P, et al. A meta-analysis of the efficacy of overthe-counter nicotine replacement. Tobacco Control 2003;12:21-7.

35 Orleans CT, Resch N, Noll E, et al. Use of transdermal nicotine in a state-level prescription plan for the elderly. A first look at 'real-world' patch users. JAMA 1994;271:601-7.

36 Haxby D, Sinclair A, Eiff MP, et al. Characteristics and perceptions of nicotine patch users. J Family Pract 1994;38:459-64.

37 Johnson RE, Stevens VJ, Hollis JF, et al. Nicotine chewing gum use in the outpatient care setting. J Family Pract 1992;34:61-5.

38 Nides MA, Rakos RF, Gonzales D, et al. Predictors of initial smoking cessation and relapse through the first 2 years of the Lung Health Study. J Consult Clin Psychol 1995:63:60-9.

39 Zhu S, Melcer T, Sun J, et al. Smoking cessation with and without assistance: a population-based analysis. Am J Prev Med 2000;18:305-11. 